\title{
Paper of October Issue of Stem Cell Reviews and Reports Presents a Novel View on Oogenesis in Adult Mammalian Ovaries
}

\author{
Sham S. Kakar ${ }^{1} \cdot$ Mariusz Z. Ratajczak ${ }^{2}$ \\ Published online: 26 August 2021 \\ (c) The Author(s), under exclusive licence to Springer Science+Business Media, LLC, part of Springer Nature 2021
}

Adult mammalian ovaries regularly form eggs that assemble as primordial follicles during adult life like spermatogenesis in adult testes. Majority of the groups working in the field have focused their research efforts on the ovarian stem cells (OSCs) which exist in large numbers and can expand in vitro and exhibit differentiation into oocytes and upon transplantation result in the birth of fertile pups. However, Bhartiya's group reported in this issue of Stem Cell Reviews and Reports two populations of stem cells including a rare, quiescent population of small sized stem cells termed VSELs along with OSCs [1,2]. The stem cells (VSELs and OSCs) in the ovaries are similar to the presence of VSELs and hematopoietic stem cells (HSCs) in the hematopoietic system [3]. VSELs in the ovaries undergo asymmetric cell divisions whereby they self-renew and give rise to slightly bigger OSCs which further differentiate into oocyte-like structures in vitro which could be physiological active. Virant-Klun's group has reported extensively on ovarian VSELs and their role in ovarian cancer [4, 5]. But how these stem cells function under normal physiological conditions, whether they undergo meiosis on regular basis in adult ovary and how these processes are influenced by circulatory hormones are questions that remain to be answered.

Sharma and Bhartiya in this issue of Stem Cell Reviews and Reports pa perused a novel approach to isolate ovary surface epithelial cells (where the stem cells are located) and carefully studied them across estrus cycle. They have successfully deciphered various events including asymmetric and symmetric cell divisions and clonal expansion of stem/progenitor cells to form germ cell nest (GCN). Various events reported in fetal ovaries were detected in the GCN in

Mariusz Z. Ratajczak

mariusz.ratajczak@louisville.edu

1 Department of Physiology, University of Louisville, Louisville, KY 40202, USA

2 Department of Medicine, University of Louisville, Louisville, KY 40202, USA adult ovary including formation of ring canals, transport of cytoplasmic organelles including mitochondria, formation of Balbiani body and meiosis. Meiosis was marked by studying the expression of SCP-3 and BrdU uptake. Interestingly, the group successfully tracked BrdU uptake in the oocyte of a secondary follicle clearly showing that stem cells have the potential to differentiate into functional oocytes under physiological conditions in vivo.

SSEA-1 positive VSELs were FACS sorted from GFP mice and transplanted in recipient Swiss mice ovaries. After two weeks, GFP and SSEA-1 positive GCN were detected which is direct proof that VSELs are indispensable in the ovaries. VSELs serve as a backup pool to give rise to OSCs throughout life. OSCs are tissue-committed progenitors with limited life-span with differentiation potential only into the oocytes whereas VSELs being more primitive and pluripotent, are immortal and possess stemness properties. This work is a significant advance and challenges existing dogma in the field and the concept of 'fixed ovarian reserve'. Thus, there is no need to worry over oocytes differentiated from VSELs versus those differentiated from OSCs. This is a linked process with VSELs role limited to undergo asymmetric cell divisions to self-renew to give rise to OSCs which differentiate further into oocytes.

More than 40 independent groups have confirmed the presence of VSELs in human tissues and being developmentally linked to primordial germ cells [3], are natural precursors for gametes, differentiate into oocytes in vitro and show a cortical reaction in presence of sperm [6]. Rather than using ES/iPS cells, scientific community may want to consider use of VSELs as a source of gametes for the infertile couples as discussed earlier [7]. Being quiescent VSELs survive oncotherapy and can be manipulated to restore ovarian function upon a healthy niche by transplanting mesenchymal stromal cells [7].

It will be interesting to study how these events in adult ovary, being reported for the first time by Sharma and Bhartiya in the present study, get altered with age, why stem cells 
stop functioning and are unable to differentiate with advancing age resulting in menopause. Also, how various insults during perinatal life affect the process of neo-oogenesis. We look forward to further progress in the field.

\section{References}

1. Parte, S., Bhartiya, D., Telang, J., Daithankar, V., Salvi, V., Zaveri, K., \& Hinduja, I. (2011). Detection, characterization, and spontaneous differentiation in vitro of very small embryonic-like putative stem cells in adult mammalian ovary. Stem Cells and Development, 20(8), 1451-1464.

2. Patel, H., Bhartiya, D., \& Parte, S. (2018). Further characterization of adult sheep ovarian stem cells and their involvement in neo-oogenesis and follicle assembly. Journal of Ovarian Research, 11(1), 3.

3. Ratajczak, M. Z., Ratajczak, J., \& Kucia, M. (2019). Very small embryonic-like stem cells (VSELs): An update and future directions. Circulation Research, 124(2), 208-210.
4. Virant-Klun, I., Zech, N., Rozman, P., Vogler, A., Cvjeticanin, B., Klemenc, P., Malicev, E., \& Meden-Vrtovec, H. (2008). Putative stem cells with an embryonic character isolated from the ovarian surface epithelium of women with no naturally present follicles and oocytes. Differentiation, 76(8), 843-856.

5. Virant-Klun, I., \& Stimpfel, M. (2016). Novel population of small tumour-initiating stem cells in the ovaries of women with borderline ovarian cancer. Scientific Reports, 6, 34730.

6. Virant-Klun, I. (2018). Functional testing of primitive oocyte-like cells developed in ovarian surface epithelium cell culture from small VSEL-like stem cells: Can they be fertilized one day? Stem Cell Reviews and Reports, 14(5), 715-721.

7. Bhartiya, D., Anand, S., Patel, H., \& Parte, S. (2017). Making gametes from alternate sources of stem cells: Past, present and future. Reproductive Biology and Endocrinology, 15(1), 89.

Publisher's Note Springer Nature remains neutral with regard to jurisdictional claims in published maps and institutional affiliations. 